\title{
Physics: Wheatstone bridge
}

\section{R Lockhat iD}

Department of Anaesthesia, Charlotte Maxeke Johannesburg Academic Hospital, University of the Witwatersrand, South Africa Corresponding author, email: dr.razzy306@gmail.com

Keywords: physics, Wheatstone bridge, unknown resistance

A Wheatstone bridge is a device which is used to find the unknown resistance. ${ }^{1,3}$ It is an instrument or a circuit consisting of four resistors or their equivalent in series which is used to determine the value of an unknown resistance when the other three resistances are known. ${ }^{1-4}$ One is variable resistance, which is also called the rheostat of the circuit, and two known resistances. It also contains the galvanometer for the detection of the current and it is also used to find the direction of current.

\section{History of the Wheatstone bridge}

Curiously enough, the Wheatstone bridge was not invented by Charles Wheatstone (1802-1875), but by Hunter Christie. ${ }^{1,3}$ However, Wheatstone was responsible for popularising the arrangement of four resistors, a battery and a galvanometer, and gave Christie full credit in his 1843 Bakerian Lecture. Wheatstone called the circuit a "Differential Resistance Measurer."1,3

To draw the Wheatstone bridge, Wheatstone himself used the familiar diamond pattern in his needle telegraph; it has been suggested that his set of Blue Willow pattern china, with the cross-hatching on the arched bridge forming part of its decorations, suggested the shape to him.

The Wheatstone bridge is well suited for the measurement of small changes in resistance. ${ }^{1-3}$ As a result, it is suitable to measure the resistance change in a strain gauge. The strain gauge transforms strain applied to it into a proportional change in resistance..$^{1-3}$ It knows these values by the way it is calibrated. Thus, if you know the voltage and can measure the current, the resistance can be calculated by using a derivation of Ohm's law; that being $\mathbf{V}=\mathbf{I R}$. From this, one can calculate the value of the unknown resistance.'

In the setting of anaesthesia, changes in resistance and current (flow) are measured. ${ }^{4}$ Based on Ohm's law, one can then deduce the resistance within the Wheatstone bridge. As a result, pressure waveforms from arterial line catheters can be converted to an electrical signal, which then are displayed as systolic, diastolic and mean arterial pressures. ${ }^{4}$

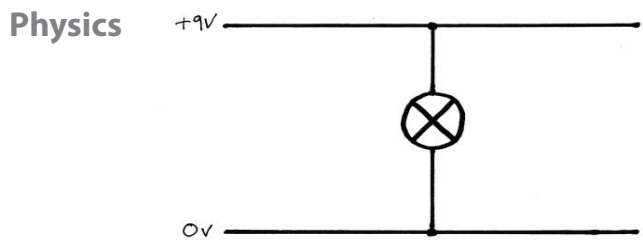

\section{Basic electrical circuit}

If we put two resistors in series, there will be a sequential drop in voltage across these resistors. At point $A$, the voltage will be the supply voltage ( $+9 \mathrm{v}$ in this case). ${ }^{4}$ At point $C$, the voltage will be Ov. Point B, however, depends on the value (number of ohms) of each of the resistors., 4 If both resistors are the same value (e.g. 100 Ohms), then the voltage at point $B$ will be exactly half the difference between points $A$ and $C$ (in this case $4.5 \mathrm{v}$ ) (Figure 2).

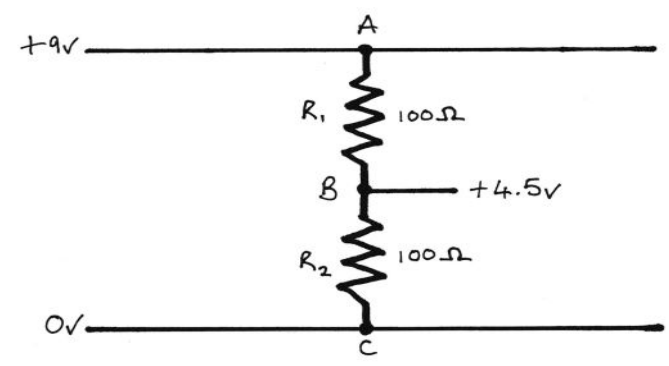

\section{Voltage divider}

Now if we change the value of resistor 1 (R1) (Figure 3) you can change the voltage at point $\mathrm{B}$. If $\mathrm{R} 1$ reduces below resistor $2^{3,4}$ (R2) then the voltage will be higher than $4.5 \mathrm{v}$, if R1 has a higher resistance than $R 2$, then the voltage at point $B$ will be lower than $4.5 \mathrm{v}$. Remember it this way, more resistance leads to more voltage drop. ${ }^{3,4}$

The voltage drop across each resistor works as a ratio to the relative resistance in each resistor. ${ }^{3}$ For instance, if we have resistor one at 200 ohms and resistor two at 100 ohms, then the ratio is $2: 1$. Hence the voltage drop across the resistors will be 2:1 (e.g. $6 \mathrm{v}$ across the first resistor and $3 \mathrm{v}$ across the second resistor). Remember the total must equal the difference between the two terminals of your power source (in this case $9 \mathrm{v}$ as in a $9 \mathrm{v}$ battery). ${ }^{3,4}$

This is called a voltage divider. It is commonly used in electrical circuits to produce different voltages than the power supply is giving you. ${ }^{1,3}$

A Wheatstone bridge is basically two voltage dividers in parallel (Figure 4). ${ }^{1,3}$ If we put two voltage dividers next to each other, and make all the resistors the same value, the voltage between 
points $B$ and $E$ will be $0 \mathrm{v}$. This is because there is no difference between the voltages at these points. ${ }^{1,3}$

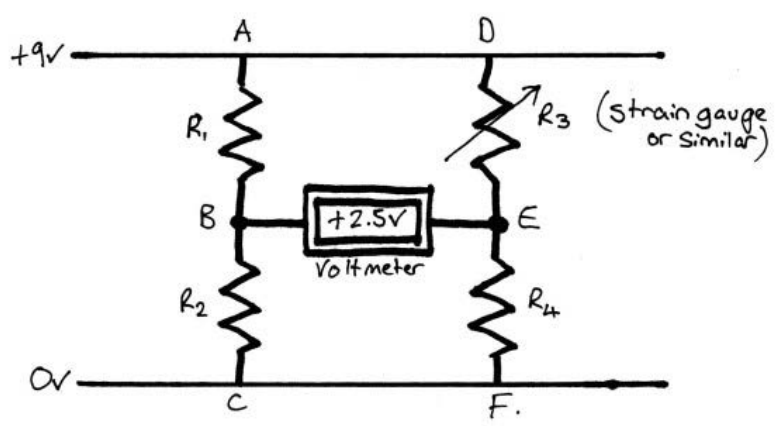

In the perioperative setting, pressure transducers convert pressure/kinetic energy into electrical energy. ${ }^{1,4}$ Pressure transducers tend to work on the principle of an increasing pressure stretching a metal element or wire. ${ }^{1,4}$ This then changes the resistance of the wire. If a wire is stretched it becomes longer and thinner and thus its resistance increases. This wire can then be connected into a Wheatstone bridge to produce a voltage in response to the pressure change. The cannula of an arterial line is connected to a pressure transducer via a column of heparinised saline at a pressure of $300 \mathrm{mmHg}$. The saline passes through a drip chamber adjusted to allow a flow of $4 \mathrm{ml} /$ hour. ${ }^{4}$ This continuously flushes the tubing and cannula. The ideal solution for use is dextrose, since a non-electrical conducting fluid avoids current passing down the catheter into the heart. This is connected to an amplifier and oscilloscope.

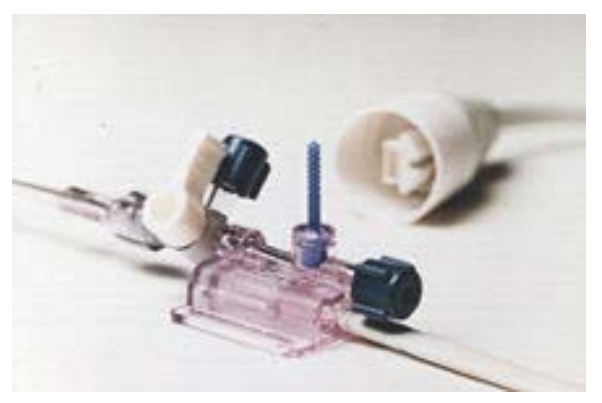

The saline column moves back and forth with the pulsation. ${ }^{4}$ This causes the diaphragm to move. This movement results in a change in resistance and current flow ( $V=I R)$ through the transducer. The transducer is connected to a Wheatstone bridge. ${ }^{4}$ The heparinised saline allows flushing of the cannula and prevents backflow. ${ }^{4}$
Newer Wheatstone bridge setups use strain gauges in all four positions. ${ }^{1,4}$ The diaphragm is attached in such a way that when pressure is applied to it, gauges on one side of the Wheatstone bridge become compressed, reducing their resistance, ${ }^{1}$ whilst the gauges on the other side are stretched, increasing their resistance. The bridge then becomes unbalanced and the potential difference generated is proportional to the pressure applied. $^{3}$

This setup of four strain gauges has the advantage that it is four times more sensitive than a single gauge Wheatstone bridge. ${ }^{2,3}$ It also compensates for any temperature change as all the strain gauges are affected equally (temperature will affect the resistance of a strain gauge so in the single gauge setup, a change in temperature will skew readings).

\section{The various uses}

Arterial pressure monitoring in perioperative settings and ICU.

It is used by electrical power distributors to accurately locate breaks in a power line.

It is also used to monitor sensor devices such as strain gauges. Such devices change their internal resistance according to the specific level of strain (or pressure, temperature, etc.), and serve as the unknown resistor $\mathrm{RX}$.

Meter bridge, post office box and Carey Foster bridge are instruments based on the principle of Wheatstone bridge. ${ }^{1,4}$

\section{Conflict of interest}

The author declares no conflict of interest.

\section{Funding source}

None.

ORCID

R Lockhat (iD https://orcid.org/0000-0002-2252-3203

\section{References}

1. Anaesthesia UK: The invasive arterial system and Wheatstone Bridge. 2004. Available from: https://www.frca.co.uk/article.aspx?articleid=336

2. Life in the fast lane: Part one. Wheatstone Bridge. Available from: https:// partone.litfl.com/wheatstone-bridge.html. Updated 2017 October 2.

3. Propofology.com: Resistor circuit analysis and Wheatstone's bridge. Available from: https://www.propofology.com/tutorials/resistor-circuit-analysiswheatstones-bridge-frca-topic. Updated 2016 March 22.

4. Jones A, Pratt $\mathrm{O}$. Anaesthesia Tutorial of the week. Physical principles of intra-arterial blood pressure measurement. Available from: http://www.e-safeanaesthesia.org/e_library/04/Physical_principles_of_intra_arterial_blood_ pressure_measurement_TOTW_137_2009.pdf. Updated 2009 June 8. 